REVISTA ANDALUZA DE ANTROPOLOGÍA

NÚMERO 16: REFLEXIONES CONTEMPORÁNEAS SOBRE ABORDAJE DE LO RELIGIOSO, EL PATRIMONIO CULTURAL Y TURISMO RELIGIOSO

MARZO DE 2019

ISSN 2174-6796

[pp. 70-92]

http://dx.doi.org/10.12795/RAA.2019.i16.04

\title{
EL SANTO QUE NO ABANDONA: SAN JUDAS TADEO EN AMÉRICA LATINA. PATRIMONIO CULTURAL Y TURISMO RELIGIOSO
}

\section{THE SAINT WHO NEVER LETS YOU DOWN: SAINT JUDES THADDEUS IN LATIN AMERICA. CULTURAL HERITAGE AND RELIGIOUS TOURISM}

Rolando Macías Rodríguez

ENAH

\section{Resumen.}

Las expresiones culturales en el siglo XXI son de los elementos que se buscan identificar y reconocer a partir de la diversidad de las culturas. Las devociones y festividades religiosas son algunas de estas expresiones que han sido identificadas como Patrimonio Cultural a nivel internacional o nacional, provocando así su consumo en perspectiva del turismo religioso. El caso que exponemos es lo acontecido en Perú de la festividad de San Judas Tadeo identificando los actores que promovieron su identificación de esta categoría a nivel nacional pero que además la misma expresión cultural no tiene reconocimiento en otros países de América Latina y sí reproducen su actividad, culturalmente hablando. A partir de los argumentos y datos presentados, identificamos los elementos de qué cultura es la que se vuelve patrimonio, quién la promueve y cómo se consume a través del turismo. 
Palabras clave. Patrimonio Cultural, festividades religiosas, turismo religioso, América Latina, San Judas Tadeo.

\section{Abstract.}

The cultural expressions in the XXI century are the elements that are sought to identify and recognize from the diversity of cultures. The devotions and religious festivities are some of these expressions that have been identified as Cultural Heritage on an international or national level, thus provoking their consumption in the perspective of religious tourism. The case that I expose is what happened in Peru of the festivity of Saint Jude Thaddeus identifying the actors that promoted their identification of this category at the national level but that also the same cultural expression has no recognition in others Latin American countries and they do reproduce their activity, culturally speaking.

From the arguments and data, we identify the elements of what culture is what becomes heritage, who promotes it and how it is consumed through tourism.

Key words. Cultural Heritage, religious festivities, religious tourism, Latin American, Saint Jude Thaddeus.

\section{Introducción}

El tema del Patrimonio Cultural ha tomado importancia a nivel jurídico en la sociedad internacional y las comunidades nacionales para ser reconocido desde las últimas décadas del siglo XX y mas aún lo que va del siglo XXI. Las discusiones conceptuales de lo que es o debe identificarse como un Patrimonio Cultural son muchísimas, aunque es de reconocer que la sociedad internacional (UNESCO) es quién predispone las particularidades nacionales de su concepto. Esta misma caracterización de ser reconocida una expresión cultural con la categoría, implica una relación entre quienes la ejecutan y quienes la consumen, haciendo más susceptible su reconocimiento a nivel social, cultural, identitaria e histórica.

En las siguientes líneas, abordamos la temática de una expresión religiosa, la devoción a San Judas Tadeo, de cómo se ha transformado en Perú, en uno Patrimonio Cultural (PC) para la nación peruana, y aunque no está considerado así en otras latitudes, si existe una manifestación cultural que podría provocar en un momento dado un reconocimiento similar. San Judas Tadeo es un símbolo perteneciente al universo sagrado del sistema religioso católico que representa una solución a las problemáticas sociales, por lo que sus devotos lo identifican, junto con la propia iglesia católica como el patrón o protector de las causas difíciles y desesperadas. En ese tenor, se utilizan diversos ejemplos en América 
Latina con los que se puede identificar dos tipologías de expresiones religiosas culturales como patrimonio: la jurídica, oficial y, la cultural, la sociedad que así lo considera.

Para llegar a lo mencionado, el trabajo está dividido en cuatro secciones, en la primera parte se discute la pertinencia del concepto cultura en la categoría PC, así como la perspectiva de importancia entre los actores que son quienes proporcionan los mecanismos para que una expresión cultural se considere en la categoría mencionada. En medio de ello, se identifica a estas expresiones como objetos culturales que serán consumidos e identificados como elementos para propiciar el turismo, uno de los objetivos que provoca en la actualidad la consideración de un PC.

En la segunda sección, se discute la particularidad de San Judas Tadeo como un elemento de doble capacidad, su característica sagrada y la social. Esto permite que los actores productores de su festividad puedan subrayar su importancia sociocultural. Posteriormente se identifica a los elementos de expresión festiva religiosa como objetos culturales en términos de producto consumible para el mantenimiento de su reproducción a nivel social interno y cultural externo. Para concluir el cuerpo del texto, se contextualiza la importancia de la celebración a San Judas Tadeo dentro de una diversidad de festividades religiosas que han sido identificadas como PC a nivel jurídico por los países de América Latina y también de algunos ejemplos que no son identificados jurídicamente pero si de manera cultural. El proceso que llevó para su estatus y los actores que participaron, desde la base, hasta conseguir ser identificado con la categoría para el caso peruano. Se finaliza el trabajo con reflexiones de lo que podrá significar la presencia de una expresión cultural a nivel local pero que se reproduce en distintas latitudes del continente que podría sugerir una extensión de la categoría a nivel cultural o incluso jurídica internacional.

\section{Cuestión inicial: ¿qué cultura es patrimonio cultural?}

Dentro de las diversas vertientes que se tienen acerca del patrimonio cultural, el cual sigue siendo uno de los temas que se encuentra en constante discusión en los diversos niveles: político, académico, social, cultural, entre otros; una de ellas es la perspectiva que en estas líneas rescatamos sobre un elemento de la cultura latinoamericana y en particular, peruana, específicamente en el contexto cultural-religioso.

En este caso, no caeremos en las discusiones tradicionales sobre qué es el patrimonio cultural, más bien, buscamos seguir una línea de discusión que versa entre la cuestión del nombramiento de los elementos culturales pero que corresponden a cierto sector de la población y por ende, de la cultura de una nación.

Dentro de América Latina encontramos innumerables elementos que han sido considerados como patrimonio cultural, ya sea material, inmaterial o como patrimonio 
natural, patrimonio histórico, y dentro de ese panorama, la cuestión del patrimonio histórico-cultural que ha construido parte esencial de lo que ahora son las naciones, pueblos y culturas de este espacio del continente americano.

En esa misma vertiente, encontramos que existe, aún más específico, un elemento que se ha construido como parte de ese patrimonio cultural, nos referimos a el patrimonio cultural inmaterial, estrictamente las expresiones religiosas, las cuales incluyen, devociones, peregrinaciones, festividades, rituales, oraciones, entre otras. Los distintos elementos del patrimonio cultural inmaterial en América Latina, presentan una diversidad de expresiones y representaciones, incluso, de similares o un mismo símbolo religioso que representa un elemento social.

La existencia de tanta diversidad de símbolos sagrados a lo largo de esta zona cultural que indicamos, en ocasiones se llegaría a pensar que no podría existir un símbolo como elemento que aglutinara a una diversidad de culturas y por ende, fuera un elemento de una zona cultural, sin embargo, de acuerdo a las investigaciones que he realizado (Macías Rodríguez, 2018), pareciera que existe un nuevo elemento simbólico sagrado que está colocando en el universo sagrado de gran parte de la población latinoamericana, este símbolo se trata de San Judas Tadeo.

A diferencia de otros símbolos religiosos, los cuales tienen una historia profunda y de participación social, además de sagrada, es preciso identificar a los nuevos símbolos que aparecen en escena de las necesidades de la población ante la incertidumbre del siglo XXI. El ejemplo de ellos, es precisamente este símbolo que analizaremos en desde esta perspectiva.

Como mencionamos, la discusión que aquí traemos está enmarca por la particularidad de hacer un análisis con base en la perspectiva de las personas, quienes son los que construyen estas manifestaciones del patrimonio cultural inmaterial y que por ende, son los que identifican las particularidades, es decir, las características. Para este caso en particular es muy importante esta postura analítica, la cual es designada como perspectiva del creyente (Masferrer, 2004: 2014), en cuanto a las expresiones religiosas y construcción de las mismas, pero sobre todo, será fundamental para entender cómo es que se desarrolla la interacción de este sector, unido al institucional (quienes designan si es un elemento cultural parte del patrimonio cultural de un país o comunidad) y la población que no es partícipe de dicho elemento cultural pero, pertenece a la misma cultura de la cual se desprenden los otros dos elementos mencionados. Esta primer hipótesis, con la que podemos designar específicamente a la construcción de un elemento cultural como patrimonio cultural, necesitamos partir de una postura de lo que es cultura.

Para esta breve discusión utilizamos el texto de Clifford Geertz (2006 ) La interpretación de las culturas, texto clásico para la perspectiva antropológica que se dedica a identificar 
la cultura como un conjunto de símbolos que los seres sociales aprenden, construyen y transmiten. En este sentido, cuando estamos identificando un elemento simbólico de este espacio, seguimos la idea del antropólogo clásico mencionado que indica que "lo que necesitamos es buscar relaciones sistemáticas entre diversos fenómenos, no identidades sustantivas entre fenómenos similares" (2006: 51). Por lo tanto, nuestra perspectiva constantemente estará dialogando entre las particularidades de un espacio de América Latina y su construcción social turística, lo cual caracteriza este proceso de construcción de patrimonio cultural. Sin embargo, en consecuencia de esta postura analítica, podemos decir que a lo que llama Geertz cultura expresa a lo que es un proceso social de construcción de las relaciones sociales, y por otro lado, lo que se identifica como patrimonio cultural, no se trata "solamente" de este nivel constructivo de símbolos que son compartidos por un conjunto de seres humanos en un espacio y tiempo determinados, es decir, es el resultado, como lo mencionábamos anteriormente, en el proceso de relaciones de una diversidad de elementos, sociedad e instituciones.

En este sentido, la perspectiva analítica que se utiliza en este trabajo se trata de la postura propuesta por José Luis García García (1998), quién identifica al patrimonio cultural como un fenómeno metacultural, es decir, el autor identifica que este proceso de patrimonio cultura se debe entender y analizar desde un aspecto histórico.

García García disgrega las diversas vertientes de cómo se construye el fenómeno del patrimonio cultural. El distingue por un lado lo que se llama "patrimonio etnológico" o "patrimonio etnográfico", en este sentido tiene una referencia desde el punto de vista del patrimonio folklorista (1998: 10). Este autor analiza la dinámica de la legalidad del proceso histórico en cómo se incorpora este elemento en la propia legislación. Si bien analiza la realidad española, lo que permite identificar en su postura es la diversidad del cómo se conforma la diversidad y particularidad cultural como un patrimonio.

Si la cultura se conforma en un objeto el cual se puede identificar como una parte de la actividad social en la que se construye una identidad partiendo de las particularidades que integran una realidad socio-cultural. Cuando se desarrolla una interacción entre un "patrimonio etnográfico" y una comunidad, población o país en la que se desenvuelve íntegramente esta expresión cultural, se construye el patrimonio cultural. En segunda instancia, esta construcción se transformará en la relación de identidad histórica y expresión actual.

Dentro de esta perspectiva, nace la necesidad de reconocer esa diversidad cultural relacionada con la identidad histórica de la acción cultural y una comunidad que la reconoce y la realiza constantemente en su preservación, para ello, una de las grandes instituciones globales, la UNESCO (United Nations Educational, Scientific and Cultural Organization) quien es un organismo especializado perteneciente a la ONU 
(Organización de las Naciones Unidas) ha desarrollado un conjunto de elementos para distinguir lo que se debe considerar como patrimonio cultural.

Una consecuencia de la expectativa de la recuperación y reconocimiento de esos patrimonios culturales, por parte de las naciones en el siglo XXI, tiene su fundamento en el desarrollo de las actividades liberales. Uno de los ejemplos es el desarrollo de la interacción entre lo público y lo privado, es decir, lo que es común a una sociedad y que no pertenece a nadie de manera personal, es susceptible de considerarse como una expresión de esa cultura. En el caso mexicano, a inicios del tercer milenio, esa relación seguía intacta, incluso la perspectiva se presentaba como bien lo analiza el antropólogo, historiador y politólogo Iván Franco:

El Marco legal que protege el PCAH [Patrimonio Cultural Antropológico e Histórico] en México y que da razón de ser al INAH [Instituto Nacional de Antropología e Historia] cuenta con una gran virtud, pero también con importantes lagunas. La primera consiste en que se establece, de forma muy clara, que la investigación es la base principal para conocer, difundir y preservar la amplia riqueza social y cultural del pasado y presente de nuestro país. La investigación, protección, conservación, restauración y recuperación de monumentos y zonas se concibe como una actividad profesional de interés social y nacional y como un labor de utilidad pública. Los intereses privados están, al menos en esta legislación, sobre patrimonio cultural (PC), explícitamente al margen (2005: 47) [subrayado propio]

Como se indica por parte del autor citado, se nota que la relación de la cultura debe de ser un elemento con cierta usabilidad por parte de la población. El uso que esa expresión cultural será considerada, posteriormente, como parte del patrimonio cultural, será la conformación de la objetivación de la cultura y puesta en el marco del mercado capitalista (desde un horizonte analítico) y otro como un reconocimiento a la diversidad de la cultura en la historia humana. Para el primer caso, lo que Iván Franco resuelve de las instancias que acontecieron en México es de consideración, ya que indica que "para los neoliberales, la cultura sólo importa en la medida en que puede ser instrumentalizada como mercancía. Usan pues, el discurso sobre cultura más como un boom o glamour cultural, lo que en los hechos sugiere que tiene un proyecto que se dirige hacia las elites y, en todo caso, de la cultura católica dominante." $(2005,101)$

Desde esta premisa, la cultura se convierte en un objeto de uso y de cambio, en ese sentido, es a lo que poco a poco se ha construido como el turismo cultural. Las diversas corrientes y discusiones que hay en torno al consumo de elementos culturales a través de la objetivación de la misma en tanto se han generado las condiciones para identificarla como un patrimonio cultural son variadas, cada punto de vista tiene que ver con la 
postura crítica de lo que se entiende como cultura, el valor intrínseco de la misma y el valor generado a partir de su unicidad y particularidad.

En consecuencia de ello, la visibilidad de los patrimonios culturales son ahora una consecuencia, también, de la propia postura del neoliberalismo y capitalismo, ideologías y perspectivas del entendimiento del mundo en donde buscan objetivizar cualquier elemento social para que sea susceptible de ser vendido, a partir de la generación de una expectativa la cual será satisfecha mediante su consumo.

Dicho argumento se obtiene de la racionalización que genera el patrimonio cultural como un elemento identificador de una sociedad, en tanto ella, la sociedad ajena a dicho grupo social buscará su consumo para reconocerla y entender el porqué de su particularidad, en tanto el propio patrimonio cultural, reafirma su distinción al ser valorada como un elemento social, una expresión humana, la cual es relevante identificar, catalogar y reafirmar como parte de una identidad social.

Para aquellos que buscan identificar el patrimonio cultural como un mecanismo de identidad social, el reconocimiento de una institución jurídica es importante. Es decir, ya que estamos caracterizando el elemento cultural, es necesario generar un halo de protección a la misma. Como bien refiere Santiago Amaya Corchuelo al identificar que en el "ámbito de la gestión política, por tanto, se incluye esta perspectiva patrimonialista que introduce nuevos parámetros ante la planificación urbanística, la protección medioambiental, las estrategias de desarrollo económico o la promoción turística" (2008: 34). La política, es decir, el Estado forma parte integral del desarrollo de este elemento de identificación, ya sea como promotor o como impulsor del patrimonio cultural para beneficio de la sociedad a la cual pertenezca dicho sector político.

Hay que tomar en cuenta, partiendo de esta perspectiva, el análisis que desarrolla Fuensanta Plata García sobre las políticas públicas respecto al patrimonio cultural en Andalucía, España. Ella expone a lo largo de su trabajo, el camino que se ha recorrido a nivel político, a través de discusiones, propuestas, designios y planteamientos políticos de cómo se debe usar la cultura como objeto y por ende el tratamiento jurídico que se le ha de realizar para su preservación y reproducción, si bien la Comunidad Autónoma Andaluza tiene poder completo sobre la cultura y su patrimonio desde 1979, la autora analiza la complejidad del tema a nivel jurídico:

La ley prevé tres tipos de inscripciones en el Catálogo General del Patrimonio Histórico Andaluz, que es el registro donde se anotan los bienes catalogados, es decir, protegidos, en Andalucía. Dichas inscripciones dependen del tipo de expediente administrativo que se tramite y tiene efectos legales diferentes para cada modalidad de inscripción, denominadas de Bienes de interés cultural, de Bienes de Inscripción Específica y de Bienes de Inscripción Genérica" (2008: 61) 
La autora antes citada, demuestra que la cultura como patrimonio es susceptible a tomar una categoría diferente a partir del tratamiento jurídico que se le otorgue. En consecuencia, cada patrimonio cultural deberá enfrentar diferentes filtros que le permitan identificarse como dignos de ser preservados y categorizados como un patrimonio cultural.

Esto mismo ocurre en el ejercicio que se realizó en la Mesa Redonda: El patrimonio cultural en el Perú y el Mundo organizada en 2013 por el comité editorial de la Revista ThemisRevista de Derecho, en donde diversos especialistas en el tema jurídico y la preservación del patrimonio cultural expusieron sus divergentes puntos de vista a cuestionamientos puntuales que se les hicieron.

Dentro de la exposición, es destacable la primer pregunta que se les hizo a los participantes para comenzar el debate, ¿qué se entiende por Patrimonio Cultural y por qué es importante preservarlo? Dado que no es la intención de estas líneas reproducir las respuestas en su totalidad del texto, sí enfocaremos la atención a la respuesta del Historiador Rafael Varón Gabal, quien contesta diciendo:

Conforme a la Ley 28296, se entiende por bien integrante del Patrimonio Cultural de la Nación toda manifestación del quehacer humano -material o inmaterialla que por su importancia, valor y significado paleontológico, arqueológico, arquitectónico, histórico, artístico, militar, social, antropológico, tradicional, religioso, etnológico, científico, tecnológico o intelectual, sea expresamente declarado como tal o sobre el que exista la presunción legal de serlo. Dichos bienes tienen la condición de propiedad pública o privada con las limitaciones que establece la citada Ley.

A fin de precisar la definición que establece la Ley peruana, es necesario considerar que también forman parte del Patrimonio Cultural de la Nación los bienes que revistan interés cultural que se encuentren comprendidos en los tratados y convenciones sobre la materia de los que el Perú sea parte. (Varón Gabai et. al., 2013: 322)

Como se puede notar, el entrevistado citado hace referencia al amplio espectro de los que se refiere la ley peruana a Patrimonio cultural, dejando abierta la posibilidad de convertirse en esa categoría toda manifestación humana, ya sea de carácter material o inmaterial. En ese sentido, tenemos entonces que la ley protege la expresión de la sociedad a la cual representa, por lo que será necesario utilizar la propia expresión del Patrimonio Cultural como una fuerza política que le de presencia y expresión a la misma sociedad que integra dicha cultura.

En el mismo ejercicio realizado por los editores de la revista ya mencionada, propusieron otra pregunta a los participantes, quienes expusieron su punto de vista al siguiente 
cuestionamiento: ¿En el Perú, cómo se procede a la valorización del Patrimonio cultura? ¿Cuál sería el modelo más adecuado? Para este cuestionamiento, nuevamente rescato la intervención del historiador quien interviene diciendo:

La declaración de un bien integrante del Patrimonio Cultural de la Nación se basa en la identificación de los diversos valores, tanto materiales cuanto inmateriales que el bien presenta, tales como el arqueológico, histórico, arquitectónico, urbanístico, artístico, tecnológico, simbólico, de identidad, así como por su autenticidad, integridad y singularidad, entre otros aspectos, los que pueden presentarse de manera individual -en algunos casos- o agrupada, pudiendo determinar incluso el grado de interés.

Una valorización del Patrimonio Cultural en términos de uso o frecuencia turística, condiciona el reconocimiento subjetivamente a la actividad comercial y las interpretaciones o intereses de las agencias u operadores turísticos que pueden orientar el interés de los usuarios, no estando necesariamente orientados a criterios protección y conservación de los bienes cuyos valores son principalmente intrínsecos. Cabe señalar que la declaración de bienes integrantes del Patrimonio Cultural de la Nación, tiene como fin el reconocimiento y protección de la herencia cultural propia del pasado de cada comunidad, que perdura en la actualidad y se transmite a las generaciones presentes y futuras, creando y/o reforzando la identidad local, regional y nacional. (Varón Gabai et. al., 2013: 330)

Para este historiador, se identifica la amalgama entre identidad, administración jurídica, expresión cultural y valor sustancial del hecho social humano, en ese sentido, construye la idea de un valor primario, la historicidad y su reproducción como particularidad en la historia de la humanidad. En ese sentido, es pertinente recuperar las palabras de Mauricio Montenegro para el caso colombiano cuando hace referencia a la pertinencia de este reconocimiento a través de las vías de las políticas actuales, mencionando:

Tal vez el giro inmaterial asociado al neoliberalismo comparta con el giro multicultural más características de las que estamos dispuestos a asumir. Después de todo, es el espacio abierto por el multiculturalismo el que ha permitido pensar (y usar) la diversidad cultural como recurso económico. Y como un recurso que exige, por supuesto, ser gestionado y administrado. ¿Cómo gestionar un recurso cuyo valor se funde en características inmateriales (tradición, autenticidad, identidad, etc.)? En las múltiples posibles respuestas a esta pregunta opera la articulación de multiculturalismo y neoliberalismo...

El papel del PCI ha sido determinante en la consolidación de un mapa global de la diversidad cultural. Por lo tanto, ha sido determinante también en la consolidación de un mercado global de la diversidad cultural. (2013: 42) 
Si la diversidad cultural ha sido calificada como un objeto cultural deseable de reconocimiento y cuidado, las legislaciones de cada país y a nivel internacional, han dado como resultado la creación de la categoría de turismo cultural. Dentro de ese turismo cultural, hay un tipo de turismo que últimamente ha tomado mucho mayor fuerza, en tanto esas expresiones culturales han sido determinadas como patrimonio cultural.

Dentro del turismo cultural, se ha desarrollado un tipo particular de este turismo, me refiero al turismo religioso. Al interior de esta categoría, podemos encontrar una diversidad de posibilidades que son considerados y catalogados como turismo cultural y patrimonio cultural. En estas líneas nos enfocamos a discutir la participación de uno de los "nuevos" patrimonios culturales en un país Latinoamericano, Perú, pero que junto con otras expresiones religiosas con las que cuenta, se enfatiza la posibilidad de considerar el territorio como susceptible de promover un turismo religioso. Si bien, solamente identificamos este ejemplo en lo particular, no es así a nivel continental, ya que la devoción a San Judas Tadeo, el caso en cuestión, aunque no es reconocido en todo el continente como parte del patrimonio cultural, si ha generado algunas intervenciones de turismo religioso. Es decir, tenemos una expresión cultural que se ha transformado en un espacio específico como patrimonio cultural y en otros tantos como parte del turismo cultural.

\section{San Judas Tadeo: Símbolo religioso y símbolo social}

Para entrar en contexto de la expresión cultural que ha sido considerada y legalizada en Perú como un patrimonio cultural, es necesario, para señalar la trascendencia de este hecho, exponemos la importancia de la devoción a este santo a nivel social y religioso, permitiendo así identificar su presencia en la cultura latinoamericana y peruana en específico.

Los aspectos que intervienen en la configuración de la realidad de la sociedad compleja, su impacto se da a distinto nivel, por un lado, el individual, por otro el cultural y finalmente en la sociedad. Cada uno de los niveles de intervención se manifiestan particularidades con mayor o menor influencia para construir sus propias individualidades que formaran vínculos a través de las características que comparten, en esta particularidad la devoción a San Judas Tadeo. Para analizar este aspecto, utilizo la categoría de santo-símbolo (Macías Rodríguez, 2018). Este concepto lo construyo y utilizo a partir de la amalgama de dos estructuras consistentes de la referencia propia de los devotos y de la sociedad externa a ellos, es decir, el individuo y su cultura. La relación con un santo, una figura religiosa, un elemento cultural es cotidiana, la gente automáticamente la identifica en los cánones de la religión católica, así como las características que intervienen en el concepto, en otras palabras, la figura del santo religioso también tiene una característica que es social en el contexto de la necesidad de poder resolver la problemática a la que se enfrentan, pero 
también cultural, una expresión que lleva a identificar a un sector poblacional como devoto de un símbolo social.

Partiendo de esta premisa obtenida a través de las entrevistas que se han realizado desde 2012 hasta 2018 en México, El Salvador, Puerto Rico, Perú, EUA, es una figura simbólica que hace milagros, dicho en otras palabras, es un agente transformador de la realidad a partir de que se le deposita la fe y el permiso (solicitud) para que intervenga en la transformación de su vida. Y al ser el continente americano un espacio geográfico en el que el sistema religioso católico fue mayoritario en todo el territorio por lo menos en cuatro siglos, la relación que se produce entre las personas y los santos es de suma importancia a nivel cultural, histórico y social. Así es lo que aconteció con San Judas Tadeo, el cual, a pesar de ser una figura sagrada que tomó relevancia en el aspecto social hasta el siglo XX y cultural en el siglo actual a nivel generalizado y público, su identificación y presencia ha tomado el mismo valor y papel que se tiene en comparación con santos tradicionales en los territorios latinoamericanos. En ese sentido:

Al identificarlo analíticamente como santo-símbolo se refiere a las referencias internas como externas del santo. En otras palabras, la figura religiosa es depositaria de un significado para las personas, sin quitarle el valor histórico-teológico que tiene, convirtiéndolo en un cúmulo de sentidos para sus vidas. No solamente es un referente del cómo se debe ser servidor o acercarse al dios católico, por el contrario, esa característica será difuminada en medida de la capacidad que tiene para transformar la realidad para sus creyentes. En dicha intervención, no se condensará únicamente el valor interno contemplativo del aspecto religioso, sino que deberá transformar las necesidades, percances, problemas, situaciones y cuestiones sociales a las cuales se enfrentan las personas en cualquier nivel socioeconómico en la vida urbana actual, en consecuencia, su intervención social y referente de la misma característica como agente de contención activo ante las necesidades sociales. (Macías Rodríguez, 2018: 356-357)

Las anotaciones hechas en la cita anterior demuestran la capacidad adaptativa del símbolo religioso que traspasa su propia calidad sagrada para trasladarse al ámbito social. Por ejemplo, para el caso puertorriqueño tenemos que la construcción -materialdel Santuario Insular de San Judas Tadeo en el sur de la isla caribeña, se necesitó el apoyo de la población, quien además fueron los impulsores de la devoción a este santo desde $1961^{1}$. Esto provocaría que la relación entre los habitantes, los clérigos y la propia figura religiosa se conectarían a nivel cultural. Esta misma relación se ve expresada en el mural

1. Véase el artículo completo en Periódico La Voz de Puerto Rico, http://nexomundo.com/vip_6/ vip_info.php?usuario=periodico\&idA=2764, consultado el 13 de septiembre de 2015 
que expresa el retablo del santuario, en el que se puede identificar a tres figuras religiosas (la Virgen de la Merced, Jesucristo y San Judas Tadeo) en medio de palmeras, garzas, flamboyanes, sociedad campesina que representa la población puertorriqueña. Esta relación le ha permitido al santo ser designado a las categorías de protector de la salud, trabajo, dinero y protección a las inclemencias del tiempo caribeño. Estas características le han permitido al santo-símbolo adquirir una referencia de turismo religioso, ya que los puertorriqueños radicados en EUA han comenzado a convivir con los devotos de San Judas Tadeo a través de murales que están en las calles de New York (Cardala \& Rodríguez, 2004; Rodríguez-Gómez, 2014).

Esta relación de los puertorriqueños han provocado un conocimiento de turismo religioso por los estadounidenses que desconocen la existencia del Santuario Insular en Puerto Rico, si bien no es uno de los principales elementos por los cuales se podría viajar a la isla, se está convirtiendo en un posible foco de turismo religioso de los devotos en el país norteamericano.

Otro ejemplo de construcción social de este símbolo, será entonces, un agente sagrado el cual, surge su existencia de una realidad diferente, si pensamos en la dicotomía sacroprofano usualmente utilizada en el análisis religioso, interviene más allá de sus facultades sagradas, generando un cambio en la sociedad visible y palpable de los devotos. En ese sentido, lo sacro se acerca a la realidad cotidiana de las personas y se convierten en símbolos significativos. En consecuencia, la denominación del capital simbólico se refleja a nivel individual y a nivel social. El reflejo de ello es la existencia de la diversidad de manifestaciones devocionales, por ejemplo en México, ya sea con peregrinaciones en caballo, bicicleta o a pie; en Puerto Rico la participación en la construcción del santuario insular, así como la cooperación económica que hacen como agradecimiento por los favores (milagros) concedidos; o el caso peruano que utiliza una institución tradicional colonial socioreligiosa por parte de los laicos, sus agradecimientos en medios de comunicación o la intervención de las interacciones en las redes sociales.

Este elemento se ve reflejado también en la actualidad social que mueve el mundo, las redes sociales y el internet. Esta expresión cultural ha tomado fuerza, ya que los datos que impactan en este sentido de incorporar al santo en el proceso actual de la cultura es cuando en 2015 el \#SanJudasTadeo se convirtió en el número uno en ser utilizado en México ${ }^{2}$. Otro de los datos a destacar es la página de la red social Facebook con mayor cantidad de seguidores de San Judas Tadeo es de 4469124 de fans, hasta agosto de 2017,

2. Véase www.tabascohoy.com/2/notas/277466/san-judas-platini-y-la-serie-mundial-en-twitter consultado el 28 de octubre de 2015 
según la web de estadística de internet socialbakers, más la cantidad enorme de páginas relacionadas al santo ${ }^{3}$.

Es entonces destacable que el elemento cultural adaptativo de este santo-símbolo tiene con mayor fuerza la cantidad de páginas de Facebook de San Judas Tadeo dos de las 10 páginas más importantes son peruanas: las cuales son dos páginas del Perú: Segunda cuadrilla San Judas Tadeo del E.A.F.C.S.J.T. con 3425 fans, estando incluso por encima de la propia página oficial de los Hermanos de El Apostolado Franciscano de Caballeros de San Judas Tadeo con 2235 fans $^{4}$.

Estos datos nos dan señal de su importancia a nivel socio-cultural. Por tal motivo, la construcción de su importancia le llevó a formar una nueva expresión cultural que sería catalogado como un patrimonio cultural.

\section{Turismo cultural y turismo religioso}

Como se indico anteriormente, la perspectiva del patrimonio cultural tiene a su interior muchos elementos que se integran como objetos culturales para posicionarse como un elemento a destacar y que se convierta en un motivo para visitar el país e insentivar el turismo cultural. En ese sentido, es importante destacar lo que se ha dado en diversos países de Latinoamérica.

Para el primer caso que podemos identificar en la dinámica del turismo religioso, tomamos en cuenta dos investigaciones a destacar para el caso mexicano, ambas realizadas por la misma autora. La primera de ellas es la realizada por Anna M. Fernández Poncela El Santo Niño de Atocha: patrimonio y turismo religioso en el cual analiza la particularidad que tiene esta figura religiosa como mecanismo de generación de una identidad en la comunidad de Fresnillo, Zacatecas en México, en la zona central del país. La autora dispone que "la identidad traspasa fronteras, y los migrantes mexicanos la llevan "del otro lado" [EUA]" (2010a: 378). Además de indicar esta migración identitaria, se construye una relación de beneficio para los propios devotos y además a los habitantes de las zonas en donde se identifica una devoción religiosa turística, "Según el Rector la gente

3. Para toda la información de los resultados véase https://www.socialbakers.com/statistics/ facebook/pages/detail/22277227650-san-judas-tadeo-el-santo-de-los-imposibles consultado el 2 de agosto de 2017

4. Las demás páginas con la palabra San Judas Tadeo y en representación se pueden consultar en https://www.socialbakers.com/search?query=san+judas+tadeo , consultado el 1 de agosto de 2017 
de Plateror vive en un " $85 \%$ del comercio generado por los peregrinos"...Patrimonio religioso-turismo-sector terciario, vocablos encadenados en el caso de esta población. Y sí, es innegable que peregrinos y turistas fomentan la actividad económica empresarial a todos los niveles y ámbitos entre los residentes del lugar" (2010a: 381). El segundo caso de la misma autora, es el de Identidad regional, fe y turismo. Nuestra señora de San Juan de los Lagos, en esta otra investigación, la autora selecciona otra figura religiosa, ahora femenina que se desarrolla en el mismo contexto socio-cultural que el anterior, el centro de México. En esta ocasión, utiliza los elementos del santuario, imagen religiosa y los milagros para ahondar en una categoría de sacralizado que llevará a transformar el territorio en un ambiente de distinción al interior y de foco turístico en el exterior planteando de la siguiente manera:

...las aparicionesy milagros delas imágenes, y los temploserigidos o reconstruidos para ellas, constituyen verdaderas identidades de los pueblos, participan de manera notable en el ordenamiento económico y social, incluso la adscripción administrativa y territorial. Construyen identidad y transforman espacios sociales; más aún, construyen o refuerzan agrego comunitario y familiar[...] Y es que los santuarios siempre tienen una influencia regional o nacional, o ambas, e incluso internacional...pero siempre de lo local hacia lo global. (Fernández Poncela, 2010b: 211-212)

En este sentido identifica la particularidad de una expresión religiosa como elemento identitario, tal como se había indicado anteriormente, pero qué sucede cuando esa expresión local, nacional tiene las mismas repercusiones a nivel internacional, dicho en otras palabras, el uso social que se le da a la expresión religiosa cultural a nivel identitario local, tiene su reflejo en otras latitudes con la misma fuerza y participación social, cultural e incluso, política. La autora citada señala que la "identidad e integración al sistema de vida comunitario, a la vida cotidiana y ceremonial. El culto y la devoción a una imagen determinada proporciona cohesión y solidaridad social, resistencia cultural identitaria, y hegemonía política y religiosa”. (Fernández Poncela, 2010b: 211-212)

Bajo este orden de ideas, rescato en ese mismo contexto un panorama que es analizado para un caso ecuatoriano por parte de José M. Lavin, Carlos Martínez-Bonilla, Franklin N. Medina-Guerrera y Walter F. Viteri-Torres quienes siguiendo el análisis del turismo religioso, identifican que en esta relación social que se hace mediada por el patrimonio cultural religioso, analizan los dos tipos de turismo que viajan con dos panoramas distintos, por un lado el elemento cultural solamente, el consumismo del mismo objeto cultural como objeto y el turismo religioso como consumidor de expresiones religiosas únicas en distintos lugares del mundo; el caso que estudian es la festividad del Señor del Terremoto en Patate (Ecuador). 
Los autores analizan la perspectiva de los tipos de turistas existentes que consumen elementos culturales-religiosos, sobre todo en una de las tres categorías que propusieron Bond, Packer y Bellantyne (2014): 1)lugares de gran valor turístico: catedrales, grandes conjuntos monumentales, es decir, objetos inmuebles; 2) lugares de peregrinación o santuarios, como los mencionados anteriormente y; 3) festivales y fiestas religiosas: eventos anuales con significación religiosa. Puntualizando la última de las tres categorías, para el caso ecuatoriano distinguirán como resultado que los perfiles del turista cultural y religioso que existen, y más aún en el caso que estudian de Ecuador, son una complejidad mayor a una sencilla dicotomía entre cultural y religioso. Esto hay que entenderlo en tanto que lo religioso forma parte fundamental de lo cultural, como lo advertimos en la primera parte de este texto con la postura de Geertz. Por lo tanto, destacamos aquí dos características que se encontraron en el caso ecuatoriano. En primera instancia "El turista religioso busca, además, compartir la experiencia con sus familiares y prefiere acompañado. La experiencia, por tanto, es colectiva también y no solamente individual... El perfil socioeconómico de clase alta del turismo cultural más típico tampoco se refleja en el turista religioso, mucho más variopinto en este aspecto (Lavín, et.al., 2017: 152).

En conclusión, la participación de diversos actores religiosos consumidores del objeto cultural (tipos de turista religioso y cultural) a pesar de tener una intensión diferente en el consumo de la expresión cultural en cuestión, se integran para compartir significados y entendimiento mediante el consumo de la expresión cultural particular. Los actores codificarán la importancia de este objeto cultural como digno de ser consumido y preservado mediante su reproducción, en consecuencia, los participantes se verán atravesados por la construcción de una identidad que se verá reflejada en un sin número de localidades, culturas, sociedades y latitudes que desconoces su existencia pero que comparten a partir de un consumo del mismo bien cultural, se conectan en una red cultural sin tener un conocimiento de la existencia de la misma.

\section{Santo, símbolo social y patrimonio cultural nacional}

Hasta este momento se han identificado diversos elementos que integran el Patrimonio Cultural, el turismo cultural y religioso como nodos de intersección del PC, los actores que participan en el consumo y la reproducción de los objetos culturales que son considerados en esta categoría. Para sintetizar todos estos elementos, analizamos el caso de San Judas Tadeo que ha sido elevado a categoría de Patrimonio Cultural de la Nación Peruana.

Es cierto que la expresión devocional a San Judas Tadeo en Perú, no es ni por cercana la primer festividad religiosa que es considerada como un patrimonio cultural, ni en el propio país, ni el único santo en otros países. Un ejemplo claro de ello es la celebración 
del Corpus Christi que se celebra en Ecuador, en al provincia de Cotopaxi, lugar en el cual se presenta una manifestación cultural de importancia tal que el sincretismo que se suscita en la danza, volcó a las autoridades para identificar la identidad y particularidad cultural de la zona (Herrera D. \& Monge, 2012: 85). La relevancia a esta festividad es tal, que ha sido nombrada como Patrimonio Cultural Intangible de la Nación en 2001 a través del Acuerdo Ministerial N. 647 ${ }^{5}$. No es el único, pues recientemente en otra parte del país, la comunidad de Alangasi, ha sido declarada como Patrimonio Cultural Inmaterial de Ecuador el 10 de junio de $2018^{6}$.

Este tipo de manifestaciones religiosas culturales, en todo el continente tienen resonancia. Si bien cada cultura tiene sus formas de expresión, es importante destacar que ellas pretenden fungir con el papel de presentación de la diversidad en la expresión particular de su cultura.

Perú es uno de los países que cuenta con la diversidad cultural en el continente americano que se destaca por su ambivalencia entre la persistencia de elementos indígenas, la formación de la cultura colonial, la diversidad de su ecosistemas y la reproducción de su cultura a partir de la presencia del catolicismo en ella. El país andino cuenta con diversos elementos culturales que han sido identificados por el Ministerio de Cultura como claves del entendimiento de la expresión religiosa particular de los peruano y al mismo tiempo, la diversidad de símbolos religiosos que coexisten en el universo sagrado de la nación heredera del Tahuantinsuyu.

El Santo católico a finales del año de 2017 se incorporó a la lista de las festividades religiosas que han sido consideradas como expresiones culturales que representan el Patrimonio Cultural del Perú, se unió a la festividad peruana católica más importante, El Señor de los Milagros, celebración que a partir del 2005 comenzó su camino a convertirse en Patrimonio cultural de la Nación, en ese año "la Resolución Directoral Nacional No 154/INC del Instituto Nacional de Cultura, y su declaración de la "Festividad del Señor de los Milagros" como Patrimonio Cultural de la Nación. La segunda el Proyecto de Ley No 4022/2009-PE, convertido en Ley No 29602, por la que se declara al Señor de los Milagros como patrono del Perú, "símbolo de religiosidad sentimiento popular" del Perú." (Benito Rodríguez \& Manjón de Garay, 2014: 25); también a la Festividad de la Virgen de la Candelaria de Chapi en el Departamento de Arequipa en la cual se identifica como una de las devociones más antiguas de la advocación mariana, decretado a través de la

5. Véase http://patrimoniocultural.gob.ec/la-fiesta-de-las-octavas-del-corpus-christi-o-deldanzante/ consultado el 12 de enero de 2019

6. Véase el certificado a dicha expresión cultural en http://patrimoniocultural.gob.ec/wp-content/uploads/2018/09/CERTIFICACION-CORPUS.png consultado el 14 de enero de 2019 
Resolución Viceministerial No 048-2012-VMPCIC-MC el 29 de agosto de $2012^{7}$. En esta misma lista, se destaca la Festividad del Señor de Locumba y la Peregrinación al Santuario del Señor de Locumba que se encuentra en el Departamento de Tacna, mediante la Resolución Viceministerial No 060-2015-VMPCIC-MC el 14 de mayo de 2015. En el mismo año, se le otorgó el mismo estatus jurídico a Festividad y Devoción al Cristo Crucificado Señor de Luren de Ica, en el Departamento de Ica como Patrimonio Cultural de la Nación, expresión religiosa que también tiene una profunda historicidad desde el siglo XVI cuando comienza su expresión devocional.

Se puede advertir a partir de los anteriores datos, que las expresiones devocionales religiosas identitarias culturales en el Perú son muchas y muy importantes a nivel loca, con la distinción del Señor de los Milagros que es a nivel nacional. Dentro de este panorama, es importante destacar que San Judas Tadeo a diferencia de las devociones anteriormente mencionadas, no tiene una profundidad histórica en su devoción de tan largo alcance como si lo son las demás. De hecho, la devoción a este santo en su expresión formal, aparece a inicios del siglo XX, cuando un grupo de transportistas que ya formaban un contingente importante en la devoción a San Judas Tadeo, gracias al impulso del Reverendo Padre Fray Orestes Alegre de la orden franciscana se constituye "La Nueva Asociación de Caballeros de San Judas Tadeo" el 28 de septiembre de 1941.

La devoción por este santo, se da en un espacio sacralizado por la religión y por ser también un Patrimonio Cultural, doblemente sacralizado, el conjunto monumental de San Francisco de Lima fue incorporado al Patrimonio Mundial de la UNESCO el 30 de diciembre de $1988^{9}$. Ahora bien, al tomar en cuenta que, Perú tiene 12 representantes culturales muebles, y uno de ellos alberga a la devoción de San Judas Tadeo, la importancia crece.

El camino para llegar a formar parte del Patrimonio Cultural de la festividad de San Judas Tadeo comenzó desde el 21 de abril de 2016, cuando el representante oficial del grupo que dirige, institucionalmentehablando, la devoción, el Presidente del Apostolado Franciscano de los Caballeros de San Judas Tadeo, el señor Gregorio Montesinos Champion y el

7. Véase la documentación oficial de la resolución en https://cdn.www.gob.pe/uploads/document/file/209917/3205.pdf consultado el 16 de enero de 2019

8. Véase https://elperuano.pe/noticia-huellas-franciscanas-73849.aspx consultado el 16 de enero de 2019

9. Véase https://presidencia.gob.do/noticias/presidente-felicita-policia-nacional-por-conmemoracion-su-patrono-san-judas-tadeo consultado el 21 de noviembre de 2018 
Secretario General, el señor Carlos Diestra Dávila solicitaron ante la Dirección General de Patrimonio Cultural, que se declarara la Festividad Procesión de San Judas Tadeo como Patrimonio Cultural. A lo largo del mismo año, se mantuvieron constantemente en búsqueda de conseguir su objetivo, el cual se lograría un año más tarde, el 18 de septiembre de 2017 mediante la Resolución Viceministerial No 173-2017-VMPCIC-MC que la solicitud hecha se hacía oficial, considerándose así la expresión religiosa como parte del Patrimonio Cultural de la Nación Peruana ${ }^{10}$. La noticia llegó tan solo un año después de que la congregación laica cumpliera sus 75 años de existencia.

Este hecho cultural del santo católico, es el único que se ha convertido en un patrimonio cultural de alguna nación, empero, no es el único lugar en donde se puede identificar la expresión devocional al mismo. En todo el continente americano, se logra vislumbrar la presencia cultural de este santo aunque no ha sido considerado como un patrimonio cultural de manera jurídica institucionalizada. Por ejemplo, tenemos que en el país caribeño de República Dominicana, el mismo santo católico es el patrón oficial protector de la Policía Nacional, de tan importancia que el propio Presidente Danilo Medina envió sus felicitaciones al órgano rector de la justicia sus felicitaciones en la celebración de su santo protector (11). También en la ciudad de Chicago, EUA, el santo es protector del mismo sector desde los años 30 , en México tiene incluso una connotación histórica de la antigua Policía Judicial a la cual se le denominaba "judas" en la cual se reconocía como miembro del sector pero también por ser devotos del mismo santo (Macías Rodríguez, 2014: 162). En la actualidad, se puede ver incluso en los sectores de altos mandos de la policía en México, haciendo celebraciones a este santo indicando su devoción y proclamación como patrono y protector (Sierra, 2013).

Esta misma situación de patronazgo y expresiones culturales de este santo, se pueden identificar en otros países del continente como en Chile donde tiene una profundidad devocional desde finales del siglo XIX (Barahona Zuleta, 2011), otro ejemplo es en Argentina donde continuamente se celebra la festividad del santo (12). Pasa lo mismo en otras latitudes, donde la celebración del santo demuestra la importancia del mismo.

Resumiendo, se puede identificar que la expresión festiva a este santo, solamente ha sido elevada a la categoría de Patrimonio Cultural en el país de Perú, sin embargo, en la mayoría de los países latinoamericanos (Macías Rodríguez, 2018) se tiene una fuerte presencia de la devoción por este santo. No se ha convertido en un elemento cultural

10. Véase https://www.aciprensa.com/noticias/como-san-judas-tadeo-contagiar-la-fe-y-el-amor-alienta-arzobispo-de-buenos-aires-85798 consultado el 19 de enero de 2019 
conocido totalmente para el turismo religioso, a excepción del caso peruano donde los connacionales llegan a viajar para la celebración del santo en octubre, empero, algunos de los representantes del Apostolado, en diversas conversaciones que tuve con ellos en el 2016, comentaban su conocimiento por la celebración en México, incluso, algunos de ellos que han tenido la oportunidad de viajar al país mencionado, buscan cumplir el objetivo de visitar tres puntos en particular, dos de carácter religioso-cultural y uno más de esparcimiento. El representante del esparcimiento es Cancún, destino paradisiaco y predilecto para vacacionar en el caribe mexicano, los otros dos son: la Basílica de Guadalupe y la llamada "Iglesia de San Judas Tadeo" (templo de San Hipólito y San Casiano), ambos recintos ubicados en la capital del país (Macías Rodríguez, 2018: 293). Así, se está convirtiendo, paulatinamente en un objeto cultural que pudiera ser considerado como un mecanismo de interacción a través de turismo religioso, ya que en México, se desarrolla la misma construcción cultural del turismo al interior del país.

\section{A modo de conclusiones}

En las líneas anteriores se ha identificado un conjunto de hechos y elementos los cuales se vienen desarrollando desde finales del siglo XX. Las cuatro secciones que componen este trabajo, se vinculan entre sí. La construcción social de la relación entre los consumidores culturales en la perspectiva turística y cultural de un patrimonio cultural, son aquellos quienes provocan que sea reconocido fuera de la misma dinámica social la propia expresión cultural.

Siguiendo la perspectiva simbólica de Geertz, los componentes de esas expresiones sirven para identificar a las comunidades en dos diferentes niveles. El primero, a nivel local, interior, es decir, los reproductores de esa expresión cultural -y por ende religiosa, como es en los ejemplos-; la otra, los consumidores turísticos de esos patrimonios culturales realizados por un conjunto de personas de la comunidad territorial o comunidad cultural. En ese sentido, los objetos culturales que han sido identificados a nivel jurídico por las instituciones internacionales y nacionales como expresiones culturales susceptibles a ser reconocidas como elementos de importancia en tanto su trascendencia y unicidad expresiva.

Como lo advertimos, para el ejemplo en particular analizado, los propios grupos sociales que realizan la expresión cultural, las festividades religiosas, son quienes solicitan ser reconocidos a partir dela profundidad histórica, colectiva, identitaria y distintiva de hecho social. A partir del reconocimiento social que representa, son susceptibles a identificarse como un Patrimonio Cultural de las naciones en específico y para la humanidad en general. Las festividades del Corpus Christi en Ecuador o el Señor de los Milagros, la Virgen de Chapi, el Señor de Luren y el propio San Judas Tadeo han sido reconocidas por los Ministerios de Cultura en cada país como esas expresiones culturales que deben 
ser consideradas como importantes. Por lo tanto, la diversidad de expresión religiosa cultural es considerada como un mecanismo de atracción turística. Al ser considerados como un objeto cultural susceptible de consumido por los turistas religiosos y culturales, la importancia de su particularidad se eleva, los valores de su significación aumentan con la finalidad de mantener su reproducibilidad. También, debemos tomar en cuenta que no todas las representaciones culturales a pesar de tener una importancia a nivel identitario, cultural e histórico significa que sean considerados como un Patrimonio Cultural de un país a nivel jurídico aunque si suceda en términos propiamente culturales, empleo de ello son el Sanyo Niño de Atocha y Nuestra Señora de San Juan de los Lagos, ambas manifestaciones en México.

En consecuencia, podemos indicar que la presencia de la devoción a San Judas Tadeo en Perú que ha sido considerada como Patrimonio Cultural y que exista la misma expresión religiosa y cultural en otras latitudes, puede ser susceptible a construir un fenómeno de turismo para aquellas personas que tengan una búsqueda de consumo religioso y cultural. Demostrando que los productores de las expresiones culturales en la actualidad, como el caso del Apostolado en Perú, son más importantes para el reconocimiento nacional o internacional para que un producto cultural sea considerado como un Patrimonio Cultural, es decir como una acción humana que representa integración de la identidad, la cultura y la historia de una sociedad. Lo que me lleva a preguntar, si las personas mismas pueden ser las promotoras de su cultura, ¿toda expresión cultural es susceptible a ser promovida como un Patrimonio cultural?, esa es una reflexión que podría ayudarnos a llevar las expresiones culturales diversas a puertos seguros en su protección, reproducción y cuidado. 


\section{REFERENCIAS BIBLIOGRÁFICAS}

Amaya Corchuelo, Santiago (2008) "Nuevas formas de gestión del patrimonio: desde la protección legal hasta el desarrollo patrimonial sostenible”. En Díaz Brenis, Elizabeth y Hernández, Javier (Coords.) Patrimonio Cultural, Turismo y Religión. México: Proa/ INAH / PROMEP, pp. 33-48

Barahona Zuleta, A. (4 de noviembre de 2011) Los Misioneros Claretianos y la devoción a S. JudasTadeo. Recuperado el 7 de marzo de 2014, de Santuario Nacional San Judas Tadeo.

Santiago de Chile: http://www.sanjudas.cl/2011/12/los-misioneros-claretianos-yladevocion-a-s-judas-tadeo/

Benito Rodríguez, José Antonio y Manjón de Garay, Sara (2014) La devoción al Señor de los Milagros. Patrimonio Inmaterial del Perú. Lima: UCSS /CEPAC

Bond, N., Packer, J. y Ballantyne, R. (2014) “Exploring Visitor Experiences, Activities and Benefits at Three Religious Tourism Sites" International journal of tourism Research, Int J. Tourism Res. Published online in Wiley Online Library (wileyonlinelibrary.com) DOI: 10.1002/jtr.2014

Cardala, Elsa B y Rodríguez, José R. (2004) “El Muralismo Religioso como Arteria Cultural de la Identidad Puertorriqueña: Voz en Contra de la Opresión” Revista Interamericana de Psicología 38(1), pp. 131-140

Fernández Poncela, Anna M. (2010a) "El Santo Niño de Atocha: patrimonio y turismo religioso" PASOS Revista de Turismo y Patrimonio Cultural 8(2), pp.375-387 http://doi. org/10.25145/j.pasos.2010.08.027

Fernández Poncela, Anna M. (2010b) "Identidad regional, fe y turismo. Nuestra Señora de San Juan de los Lagos”. En Hernández López, J. , Rotman, M. B. y González de Castells, A. N. (2010) Patrimonio y cultura en América Latina: nuevas vinculaciones con el estado, el mercado y el turismo y sus perspectivas actuales. México: Universidad de Guadalajara, pp. 207-234

Franco, Ivan (2005) Transformaciones del proyecto cultural en México. Educación, cultura y patrimonio cultural ante el neoliberalismo, México: Delegación DII-IA-! Sección 10 SNTE

García García, José Luis (1998) “De la cultura como patrimonio al patrimonio cultural”. Política y Sociedad 27. Madrid: Universidad Complutense de Madrid, pp. 9-20

Geertz, Clifford (2006) La interpretación de las culturas. Barcelona: Gedisa 
Herrera D., Sylvia y Monge, Elena (2012) “Corpus Christi, Patrimonio Intangible del Ecuador”. RICIT 4, (octubre): Universidad de Especialidades Turísticas, pp. 71-86

Lavín, José M. et. al. (2017) “Diferencias entre el perfil del turista cultural y el turista religioso. La festividad del Señor del Terremoto en Patate (Ecuador)" Methodos. Revista de Ciencias Sociales, 5 (1), pp. 142-154 http://dx.doi.org/10.17502/m.rcs.v5i1.159

Macías Rodríguez, Rolando (2018) "Hace posible lo imposible" El señor de las causas desesperadas. San Judas Tadeo en América Latina (Tesis de doctorado), México: ENAH

Masferrer Kan, Elio (2014) Religión, política y metodologías. Aportes al estudio de los sistemas religiosos. México: Libros de la Araucaria

Masferrer Kan, Elio (2004) ¿Es del César o es de Dios? Un modelo antropológico del campo religioso. México: IIS/ UNAM / Plaza y Valdés

Montenegro, Mauricio (2014) Articulaciones entre políticas económicas y políticas culturales en Colombia. El patrimonio cultural, el sector artesanal y las nuevas formas del valor y la propiedad. Boletín de Antropología 28(46). Medellin: Universidad de Antioquía, pp. 35-52. Recuperado de http://aprendeenlinea.udea.edu.co/revistas/index. php/boletin/article/view/19519/16439

Plata García, Fuensanta (2008) "Algunas reflexiones acerca del patrimonio y las políticas públicas en Andalucía” en Díaz Brenis, E. \& Hernández, J. (Coords.) Patrimonio Cultural, Turismo y Religión. México: Proa/ INAH / PROMEP, pp. 49-67

Rodríguez-Gómez, José (2014) "Murales urbanos: amonestación en contra de la violencia” Revista de Ciencias Sociales 27, pp. 196-213

Sierra, A. (29 de octubre de 2013). “El día de los buenos muchachos”. Periódico El Reforma.

Varón Gabal, R. et. al. (2013) "El Patrimonio Cultural en el Perú y el Mundo" en ThemisRevista de Derecho 63. Perú: PUCP, pp. 321-341

\section{CIBERGRAFÍA}

Como San Judas Tadeo contagiar la fe y el amor: alienta Arzobispo de Buenos Aires: https://www.aciprensa.com/noticias/como-san-judas-tadeo-contagiar-la-fe-y-el-amoralienta-arzobispo-de-buenos-aires-85798 [consultado el 19 de enero de 2019]

Noticia oficial del recordatorio de los 30 años de la categoría de Patrimonio Mundial de la Humanidad del conjunto monumental del convento de San Francisco: https://elperuano. pe/noticia-huellas-franciscanas-73849.aspx [consultado el 16 de enero de 2019] 
Documento oficial de Resolución Viceministerial del Perú sobre la festividad del Señor de Locumba: https://cdn.www.gob.pe/uploads/document/file/209609/rvm060.pdf [consultado el 16 de enero de 2019]

Documento oficial de Resolución Viceministerial del Perú sobre la festividad de la Virgen de la Candelaria de Chapi: https://cdn.www.gob.pe/uploads/document/file/209917/3205. pdf [consultado el 16 de enero de 2019]

Documento oficial de Resolución Viceministerial del Perú sobre la festividad de San Judas Tadeo: https://cdn.www.gob.pe/uploads/document/file/209147/rvm173.pdf [consultado el 15 de enero de 2019]

Certificado original del Estado Ecuatoriano sobre el patrimonio inmaterial de la festividad del Corpus Christi en Alangasi: http://patrimoniocultural.gob.ec/wp-content/ uploads/2018/09/CERTIFICACION-CORPUS.png [consultado el 14 de enero de 2019]

Documento original del Estado Ecuatoriano sobre el patrimonio cultural inmaterial de la festividad de las octavas del Corpus Christi en Cotopaxi: http://patrimoniocultural. gob.ec/la-fiesta-de-las-octavas-del-corpus-christi-o-del-danzante/ [consultado el 12 de enero de 2019]

Presidente felicita a policía nacional por conmemoración de su patrono San Judas Tadeo: https://presidencia.gob.do/noticias/presidente-felicita-policia-nacional-porconmemoracion-su-patrono-san-judas-tadeo [consultado el 21 de noviembre de 2018]

Resultados completos de la búsqueda en Socialbakers de la palabra San Judas Tadeo: https://www.socialbakers.com/statistics/facebook/pages/detail/22277227650-san-judastadeo-el-santo-de-los-imposibles [consultado el 2 de agosto de 2017]

Resultados de búsqueda de la palabra San Judas Tadeo en la red social Facebook se pueden consultar en https://www.socialbakers.com/search?query=san+judas+tadeo [consultado el 1 de agosto de 2017]

Artículo periodístico de $\mathrm{La} \mathrm{Voz}$ de Puerto Rico sobre la construcción del Santuario Insular de San Judas Tadeo: http://nexomundo.com/vip 6/vip info. php? usuario=periodico\&id $A=2764$ [consultado el 13 de septiembre de 2015]

Nota periodística de las tendencias en Twitter del periódico mexicano Tabasco Hoy: www.tabascohoy.com/2/notas/277466/san-judas-platini-y-la-serie-mundial-en-twitter [consultado el 28 de octubre de 2015] 